\title{
Streamlining Offload Computing to High Performance Architectures
}

\author{
Mark Purcell ${ }^{1}$, Owen Callanan ${ }^{1}$, and David Gregg ${ }^{2}$ \\ 1 Dublin Software Lab, IBM Ireland \\ 2 Trinity College Dublin, Ireland \\ \{mark_purcell, owen.callanan\}@ie.ibm.com, david.gregg@cs.tcd.ie
}

\begin{abstract}
Many important scientific, engineering and financial applications can benefit from offloading computation to emerging parallel systems, such as the Cell Broadband Engine ${ }^{\mathrm{TM}}$ (Cell/B.E.). However, traditional remote procedure call (RPC) mechanisms require significant investment of time and effort to rewrite applications to use a specific $\mathrm{RPC}$ system. As a result, offloading functions to remote systems is not viable for many applications. IBM ${ }^{\circledR}$ Dynamic Application Virtualization ${ }^{\mathrm{TM}}$ (DAV) insulates the application developer by automatically generating stub libraries that allow direct calling of remote procedures without application source code modification. In this paper, we describe DAV automates the conversion of client applications to use remote procedure calls. DAV can generate stub libraries for a wide variety of client applications running on a variety of architectures, allowing allows simple and fast remote procedure call enablement of applications with minimum programming effort.
\end{abstract}

Keywords: Offload computing, computer architecture, parallel computing, remote procedure call.

\section{Introduction}

Offloading computation onto remote computer systems is a useful way to accelerate computationally intensive applications. For example, in the financial services sector, spreadsheet applications are used to evaluate options prices using the Black-Scholes formula. This is a computationally intensive algorithm which can be significantly accelerated on specialised processors such as the Cell/B.E. 2]. Offloading the Black-Scholes formula onto remote high performance systems significantly improves performance of the spreadsheet, allowing faster response to financial market conditions. Offloading calculations from the application to the remote library is a significant challenge in the industrial setting however.

Traditional remote procedure call (RPC) mechanisms enable applications to call functions from libraries running on remote machines. These systems require the client application to use a specific API, forcing an application rewrite. The time and effort required is significant and, as a result, offloading functions using traditional remote procedure call mechanisms is often not viable. Such RPC 
mechanisms can also create maintenance problems since changes in the API require rewriting the client applications. In the industrial domain, these problems are a major barrier to offloading computation to systems optimised for particular types of processing, such as machines based on the Cell/B.E. processor, which is heavily optimised for numerical computing [2].

The Virtualizer component of IBM Dynamic Application Virtualization (DAV) addresses this problem. Based on library and function specific tags supplied by the library developer, the Virtualizer generates libraries that exactly mimic the interface of the local machine libraries. As a result, no application code changes are required to offload functions to remote machines using DAV. The client application need only relink to the Virtualizer generated libraries, instead of the native code libraries. The Virtualizer currently generates stub libraries C, C++ and Java stub libraries. These stub libraries are generated from simple tags in a small domainspecific language. Furthermore DAV can be extended to support other languages and applications if required.

This paper presents an overview of DAV and the Virtualizer tool and we discuss the efficacy of using generative code techniques to eliminate application code changes when offloading functions to remote machines. The paper starts with an overview of the DAVsystem in section 2.1. Section 3 then continues with a discussion of the problems faced in automatically generating libraries that handle variables and function parameters with unknown size and usage. In section 4 we describe how Virtualizer uses a simple domain-specific language to describe the size and usage of parameters, and a generator to automatically generate interface code for remote procedure calls. Finally in section [5, a brief discussion of a traditional remote procedure call system, RPCGen, is included.

\section{DAV Overview}

\subsection{DAV Architecture}

The default DAV data transport system provides a flexible, extendable, light weight, low-latency infrastructure to allow client applications to access libraries on remote systems, giving clients access to multiple remote libraries, or services, through a central broker. Services register availability with the broker, which then maintains information on the available services. To increase availability of a service, multiple instances of a service can be run concurrently on multiple machines. In order to access an DAV service, client applications send a service request to the broker and the broker then chooses the best available service node to process the request. The client processes its service request by interacting directly with this service node. DAV services can run on various high performance architectures including x86 processors and the Cell/B.E. processor. Thus the broker provides a single interface to multiple services, running on multiple nodes with heterogeneous architectures.

DAV can use other transport systems. For example, DAV could use a third party grid scheduling system. This is possible since DAV defines a simple interface API. 


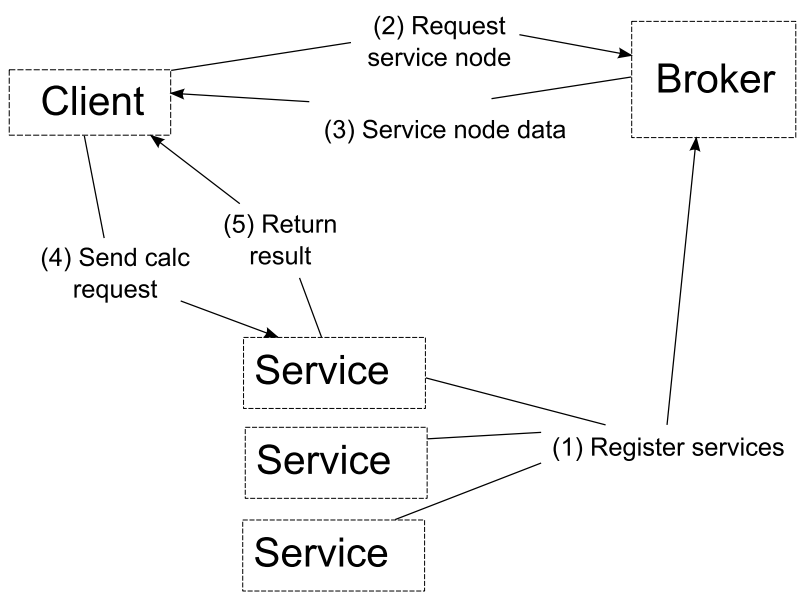

Fig. 1. DAV Architecture

Any transport subsystem that implements this API can be used by DAV. By implementing a wrapper interface for this grid scheduling system, an organisation can use DAV to allow their applications to easily offload calculation to remote machines, whilst utilising their investment in their existing grid infrastructure.

The DAV code generator generates client-side stub libraries that duplicate the interface of the original native code libraries. These libraries enable client applications to call remote library functions without changing the application source code. For an application to use DAV services, the library developer must write tags, which are included in the library header file, for all the functions in the library to be offloaded. The Virtualizer takes the modified header file as input and uses the DAV tags to generate client-side stub libraries and server-side skeleton libraries. The server-side skeleton libraries are automatically deployed to the remote server using the DAV deployment tool. To access the offloaded library, the client application need only be linked to the generated client stub libraries instead of to the original native code libraries. Once the DAV service has been started and registered with the broker, the offloaded library is available for use.

Traditional function offload systems require that the client application is rewritten to use a specific API; a process which is often difficult and timeconsuming. The resulting code can be difficult to maintain since changes to the API require rewriting of the client application. Automatically generating libraries that handle all communication to and from the DAV services addresses these problems, making DAV-enabled applications easy to use and maintain. If the DAV infrastructure changes then the developer need only regenerate the stub libraries and relink the application.

\subsection{DAV Application Areas}

Performance is is often limited by the hardware platform running an application. For example, a spreadsheet performing computationally intensive data analysis 
is limited by the desktop computer it is run on. Meanwhile, specialised high performance computing systems such as the Cell/B.E. processor can deliver significant performance improvements over standard processors for many common computing kernels 38. Larger multiple processor machines such as clusters of x86 or Cell/B.E. processors now provide significant processing power and DAV offers an excellent solution to accessing the performance of these systems from existing applications.

DAV is initially targeted at the financial services sector, but it has applications in other fields of business, science and engineering. For example many science and engineering applications are based on standard linear algebra packages such as BLAS 4. The Cell/B.E. delivers significant performance for many of the core BLAS routines, and DAV provides a straightforward way to access this performance.

\section{RPC Challenges}

Many languages, including $\mathrm{C}$, do not have self-describing data structures. Given a pointer to a data structure, the language doesn't know the size or shape of the data structure. Pass-by-reference array parameters are an example of this problem. Unless the size of the array is known at compile time, then extra information must be passed to the function to indicate the size of the array. An example of this is shown in figure 2. Unknown parameter sizes are a problem for remote procedure offload systems such as DAV, since the operand data must be transferred to the remote server and unless the size of the data is known, data transfer is not possible. Thus some mechanism is required to specify the size of pass-by-reference parameters to allow DAV to generate correct stub libraries.

Pass-by-reference parameters can also be modified within a function, or a function may allocate memory to a pointer passed as a parameter. As a result, operand data may only need to be sent, retrieved, or sent and retrieved. The $\mathrm{C}$ language syntax does not specify how a pointer passed to a function is used inside the function and, to maximise efficiency, data should only be transferred when necessary. Again, a mechanism is required to specify the transfer direction of pass-by-reference parameters.

\section{A Solution: DAV Tags}

DAV tags provide a solution to automatic transfer of pass-by-reference parameters. Using these tags, the library developer can specify that a function is to be hosted in the remote library, and the size and transfer direction of any pass-by-reference parameters. DAV tags are based on the DOxygen/JavaDoc tags used to specify documentation information within application source code. DAV tags can also pass other information to the Virtualizer, such as the library name or information about structs. The format of the DAV tags is as follows: 


\section{$/ * *$ IBMDAV* \\ @tagType value \\ Qproperty value \\ Oproperty value \\ ...* *}

There are three main tag types:

1. Library tags specify settings for the entire library including adding prefixes or suffixes to DAV exported functions.

2. Function tags set properties for specific functions, including the size and transfer direction of pass-by-reference parameters and return values.

3. Struct tags are used to inform the Virtualizer about any structs used by DAV exported functions, including the size of any pointer type struct members.

Various property tags are used by the three tag types, and are shown in table 1 .

Table 1. DAV semantic property tags

\begin{tabular}{|c|l|c|}
\hline Property Tag & Purpose & Used with \\
\hline \hline @library $<$ name $>$ & Library name & - \\
\hline @func $<$ name $>$ & Virtualize function $<$ name $>$ & - \\
\hline @struct $<$ name $>$ & Describe struct $<$ name $>$ & - \\
\hline @param[in|out|inout] $<$ name $>$ & Function-param details & @func, @struct \\
\hline @return & Specify size of returned data & @func \\
\hline @dimensions [<size $>$ ] & Specify size of an array & @param, @return \\
\hline @ type string & Denote string parameter & @param, @return \\
\hline @prefix $<$ p $>$, @suffix $<$ s $>$ & Function prefix or suffix & @library \\
\hline @lib_options " $<$ options $>$ " & Additional linker options & @library \\
\hline
\end{tabular}

Figure 2 shows a $\mathrm{C}$ function that takes two pointers to arrays as operands, along with an integer to specify the size of the arrays. Also shown in figure 2 are the tags required for the Virtualizer to create stub libraries for the function. The Virtualizer handles the integer parameter $s$, automatically because it is a scalar type, but extra information is required for the two pointer parameters to describe the data that they point to. In this case, they are both pointers to arrays of size $s$. The first array, $a$, is input only, whilst the second, $z$, is both an input and an output since it is modified by the function. The@param and @ dimensions tags are used to pass this information to the Virtualizer.

Figure 3 shows the code generation process used by the Virtualizer. The user runs the Virtualizer from the command line, passing the library header file including tags, as input. The Virtualizer checks the syntax of the $\mathrm{C}$ header definitions and of the tags, and parses the header file using the Eclipse $\mathrm{C} / \mathrm{C}++$ parser, CDT. Function prototype information is extracted and stored in a temporary internal representation. The tags are extracted from the header file using 


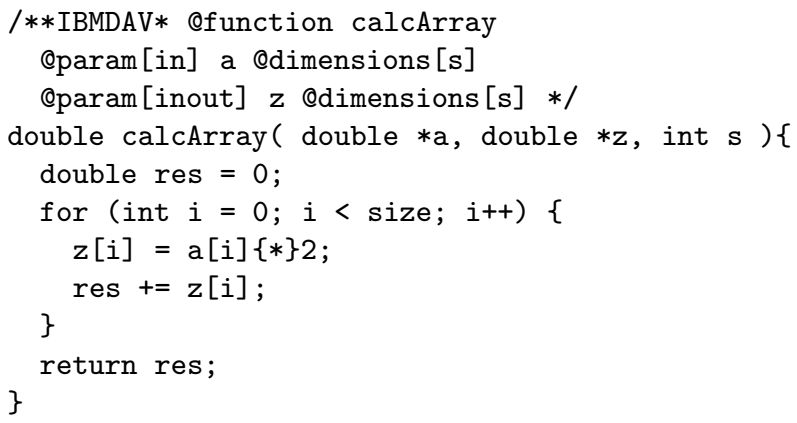

Fig. 2. Function with DAV tags for unknown parameter size and transfer direction

CDT and passed to the Java parser-generator, Javacc, which extracts the information from the tags and stores it. The Virtualizer processes the stored tag and the header file information, combining them into a single XML document. Using XSLTs, the Virtualizer produces the source code for both client-side stub libraries and server-side skeleton libraries. Finally the client-side stub library is compiled on the client system. The server-side skeleton library is then deployed to the machine that will run the service using the DAV deployer tool.

When run using the tag information shown in figure 2, the Virtualizer will produce a client-side stub library that exports a calcArray function with identical syntax to the original native code function. This function consists of code to construct the transportable data, manage calling of the remote DAV function and extract the returned result data. All interactions with the underlying infrastructure are completely contained by the generated function, so no code changes to the client application source are required.

By using generative code techniques DAV supports a variety of client types including $\mathrm{C}, \mathrm{C}++$, Java and VBA applications. $\mathrm{C} / \mathrm{C}++$ are supported for server side libraries. All standard basic types in each language are supported as well as strings, arrays, two dimensional arrays and data structures. Pointers to all of these types, including pointers to arrays of up to two dimensions, are also supported. All supported types are natively supported, no DAV specific types are required, so DAV requires no client side code changes of any kind.

\section{Related Work}

RPCGen is an example of a traditional API based RPC mechanism [5]. RPCGen simplifies the use of RPC by generating stub libraries that wrap much of the RPC API. However RPCGen still requires significant code changes to the client application. RPCGen remote procedures use a different function call syntax to the original native code functions, and some parameter types, such as variable length arrays, must use RPC specific data types instead of the native $\mathrm{C}$ types. As a result the client application code must be changed to allow RPCGen remote procedures to be used. A significant amount of work is required to convert 


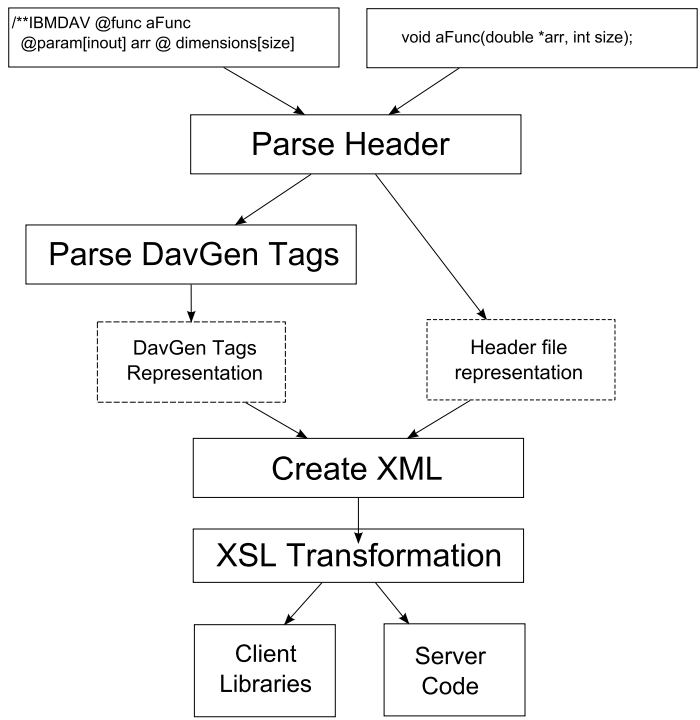

Fig. 3. Code generation process

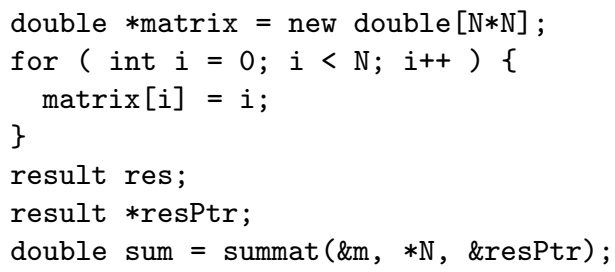

Fig. 4. Original Native Source Code

an application to use RPCGen remote procedures, as shown by comparing the original source in figure 4 with the source modified to use RPCGen in figure 5 .

CORBA is an object request broker system that allow applications written in different languages to exchange data objects [1. CORBA uses a languageindependent interface definition language (IDL) to define the interface between client applications and the object broker system. This interface code is then compiled into language-specific stub and skeleton libraries using the IDL compiler provided with a CORBA implementation. The use of an interface definition language allows CORBA implementations to insulate the application developer from some of the complexity of the CORBA object broker system. However substantial code changes are still required, as can be seen in figure 6. Component Object Model (COM) from Microsoft is another technology that allows different applications to interact though a common object format [6]. In a similar fashion to CORBA, COM uses an IDL to define the interface between applications and the COM data transport system. 


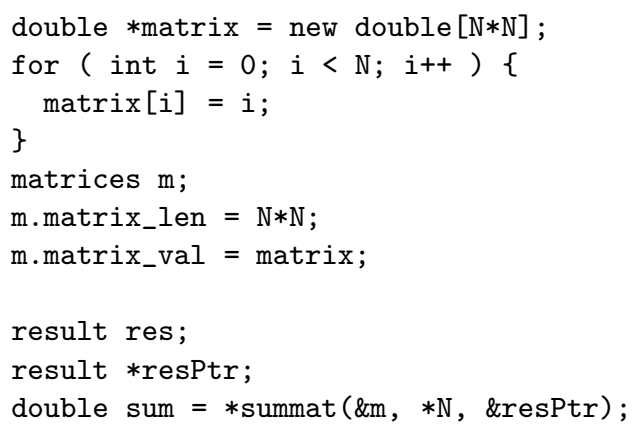

Fig. 5. Example of use of RPCGen specific types for $2 \mathrm{~d}$ matrix function

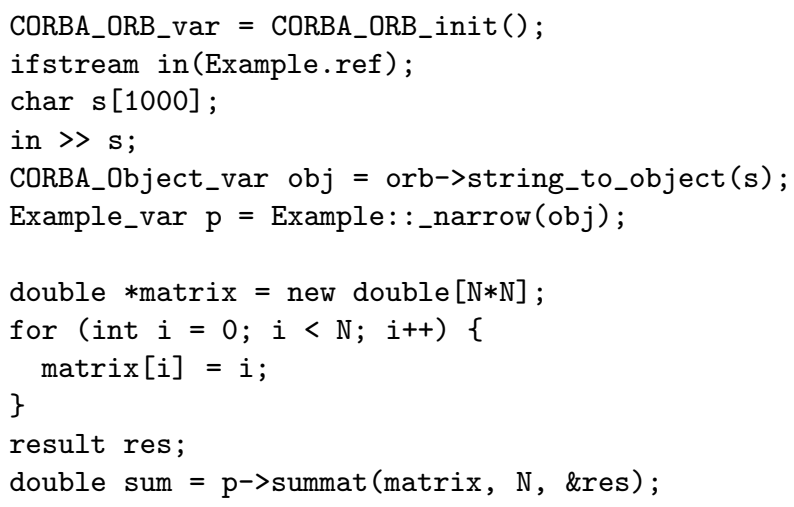

Fig. 6. Example of CORBA code changes for a $2 \mathrm{~d}$ matrix function

Automatically partitioning an application to distribute it across multiple systems is a related problem to the one we address. An example is J-Orchestra [7. which can automatically partition and distribute Java bytecode programs to execute just as if they were running on a single system. J-Orchestra works by rewriting the Java bytecode to insert an extra level of indirection into all object references. By intercepting memory accesses in this way, references to objects on remote systems can be redirected, and similarly local method invocations can be replaced by remote procedure calls. It is important to note that J-Orchestra only works because Java has well-behaved references and self-describing data structures. Redirecting all memory references would be much more difficult in $\mathrm{C} / \mathrm{C}++$ because general pointers can be used to access arbitrary parts of memory.

\section{Experimental Results}

To maximise the performance gain from calculation offload to a remote system using DAV, the offload overhead must be minimised. There are two main causes 
Table 2. DAV latency performance data

\begin{tabular}{|l|l|}
\hline \multicolumn{1}{|c|}{ Performance Test } & Latency $(\mu \mathrm{s})$ \\
\hline Local Transport & 59.9 \\
\hline DAV Transport (localhost interface) & 260 \\
\hline DAV Transport (remote server, Gigabit Ethernet) & 406 \\
\hline
\end{tabular}

of overhead in an DAV request. The first is the data marshalling overhead which is the time required to pack and unpack request and result data into a transportable format. The second overhead is the cost of transporting the data to the remote system. To measure the data marshalling overhead, a different transport subsystem is used, which is called the local transport. The local transport is a skeleton transport that processes requests on the client machine, and does not use the network stack at all. Measuring the time required to process requests using the local transport allows measurement of the calculation overhead from using DAV to process requests. The network stack overhead is measured using the standard DAV transport by running the client and server on the same machine, accessing the server through the network stack localhost interface. Finally the latency is measured for a client accessing a remote service over a typical network. Latency figures are presented for these three scenarios. The test function used is shown in figure 2. The operand arrays contain 4 elements each. As a result the time required to execute the test function is very small compared to the overall time required to process a remote request. The client machine is a $2.4 \mathrm{GHz}$ Intel Core 2 processor running Windows, and the server machine is a $3.0 \mathrm{GHz}$ Intel Xeon processor running Linux.

The performance figures in table 2 show that processing required for a single small DAV request is low, and overall takes less than 60 microseconds. Processing an DAV request using a service running on the client machine, accessed through the localhost network interface adds a further 200 microseconds to this figure. This reflects the increased processing overhead caused by moving data through the network stack. Finally, processing the DAV request over a Gigbit Ethernet network takes a little over 400 microseconds, which shows the cost of transporting the request and result data across the network.

Functions with high computational complexity are easier to accelerate, since the offload overhead is small compared to calculation time. For large data volumes, the speed of the network system is the critical factor in determining offload overhead, since the cost of making the remote function call is small compared to the cost of transporting the data. For small data volumes, where the data transport cost is low, the cost of the function call is comparitvely large. We can see from the above figures that DAV has a low function call overhead, allowing DAV to accelerate even relatively small calculations.

\section{Conclusion}

Traditional RPC mechanisms require significant application code changes to offload functions onto remote machines. This a major barrier to adoption of offload 
computing in industry, because customers are reluctant to significantly modify their software. DAV's Virtualizer tool addresses this issue and eliminates any application code changes when converting to remote procedure calls, substantially simplifying the process of converting an application. The Virtualizer uses a straightforward set of tags to allow the developer to supply information about unknown variables and function parameters such as pointers to arrays. When supplied with these tags in the library header file, the Virtualizer generates source code for client stub libraries and server skeleton libraries that wrap all interactions with the DAV infrastructure, completely insulating the client application. The external interface of the client-side stub library is identical to the original native code library, so no application code changes are required to enable an application to use remote procedures. Furthermore the client application is protected from any changes to the middleware API. If the API changes, then the source code for the libraries need only be regenerated, and the application rebuilt using the new libraries. This greatly reduces the effort required by industrial users of DAV to offload computations from the desktop to more powerful cluster computers. The use of domain-specific generative code techniques allows DAV to substantially reduce the cost and effort required to write and maintain applications that offload calculation to remote systems.

\section{References}

1. Common object request broker architecture: Core specification. Technical report, Object Management Group (2004)

2. Kahle, J.A., Day, M.N., Hofstee, H.P., Johns, C.R., Maeurer, T.R., Shippy, D.: Introduction to the cell multiprocessor. IBM J. Res. Dev. 49(4/5), 589-604 (2005)

3. Kurzak, J., Buttari, A., Dongarra, J.: Solving Systems of Linear Equations on the CELL Processor Using Cholesky Factorization-LAPACK Working Note 184. LAPACK Working Note 184 (May 10, 2007)

4. Lawson, C.L., Hanson, R.J., Kincaid, D.R., Krogh, F.T.: Basic linear algebra subprograms for fortran usage. ACM Trans. Math. Softw. 5(3), 308-323 (1979)

5. Rago, S.A.: UNIX System V network programming. Addison-Wesley Longman Publishing Co., Inc., Boston (1993)

6. Rogerson, D.: Inside COM. Microsoft Press, Redmond (1997)

7. Tilevich, E., Smaragdakis, Y.: J-orchestra: Automatic java application partitioning, pp. 178-204. Springer, Heidelberg (2002)

8. Williams, S., Shalf, J., Oliker, L., Kamil, S., Husbands, P., Yelick, K.: Scientific computing kernels on the cell processor. International Journal of Parallel Programming 35(3), 263-298 (2007) 